\section{Banking on ATM}

Huntington disease (HD) is a debilitating fatal progressive neurodegenerative disorder that is currently untreatable. It is caused by a trinucleotide expansion in the gene encoding huntingtin (HTT), resulting in the formation of mutant huntingtin (mHTT), but the molecular mechanisms underlying HD pathogenesis remain poorly understood. Now, reporting in Science Translational Medicine, Yang and colleagues show that ataxia telangiectasia mutated protein (ATM), a central regulator of the DNA damage response, may be a promising new target in HD.

The DNA damage response is consistently upregulated in agerelated neurodegenerative diseases such as Alzheimer disease and HD. ATM, a PI3K-like kinase, is activated by DNA damage and

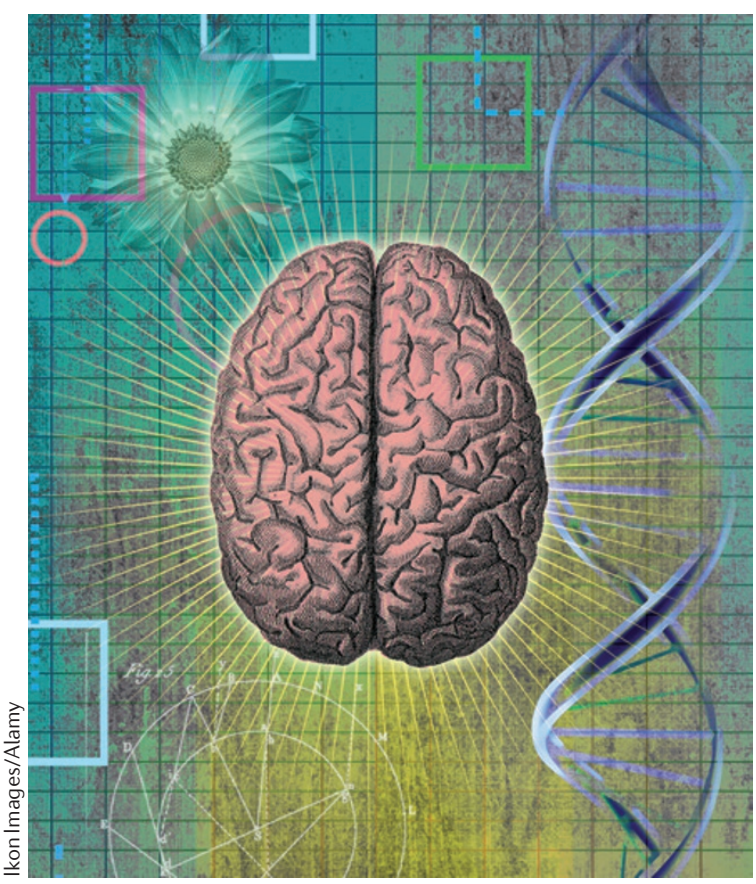

oxidative stress, and phosphorylates key molecules at the DNA damage site. It can also induce apoptosis via the activation of p53. Individuals with two mutated ATM alleles suffer from ataxia telangiectasia, a disorder involving cerebellar neurodegeneration, immunodeficiency and cancer susceptibility. However, heterozygous carriers seem to be unaffected.

In $\mathrm{HD}$, the earliest and most profound degeneration occurs in the striatum, and previous studies have shown enhanced activation of ATM in striatal neurons in patients with HD. To investigate whether ATM plays an active part in the pathology of HD, the authors first used a model of oxidative-stress-induced DNA damage in mouse embryonic fibroblasts that were derived from a transgenic HD mouse model expressing full-length human mHTT. In this model, the presence of mHTT significantly enhanced ATM activation in response to oxidative stress, leaving these transfected cells more vulnerable to stress-induced cell death. Additionally, in a model of mHTT-fragment-induced cell death in human neuroblastoma cells, short hairpin RNA-mediated knockdown of ATM protected against cell death.

Next, using a Drosophila melanogaster model of $\mathrm{HD}$, the authors found that flies expressing a null allele of the ATM homologue tefu had significantly reduced motor defects compared with their tefu $u^{+/+}$counterparts. Moreover, in a transgenic mHTT mouse model of HD, mice that had one Atm null allele performed notably better in a well-established battery of motor and behavioural tests than did $\mathrm{Atm}^{+/+} \mathrm{HD}$ mice, indicating that HD pathology can be partly ameliorated by reducing ATM gene dosage.
To investigate whether pharmacological inhibition of ATM improves HD phenotypes, smallmolecule inhibitors of ATM were tested in mHTT-transfected primary rat corticostriatal co-cultures. Treatment of these cells with KU-60019, a potent and specific small-molecule inhibitor of ATM, resulted in protection of striatal neurons from mHTT-induced toxicity. A similarly protective role of ATM inhibition was shown in neuronal cells differentiated from two induced pluripotent stem cell (iPSC) lines derived from patients with HD. These cells showed various mHTT-related phenotypes and, unlike neuronal cells derived from control iPSC lines, were dependent on the presence of brain-derived neurotrophic factor (BDNF). The addition of KU-60019 provided protection from BDNF-withdrawalinduced cell death of HD iPSCderived mixed-lineage neuronal cells, but had no effect on neuronal cells derived from control iPSCs.

The authors stress that it is still unclear how mHTT causes aberrantly elevated ATM signalling, how ATM inhibition mediates neuroprotection, and whether chronic ATM inhibition would be feasible. However, given that heterozygous ATM mutation carriers are healthy, and that caffeine, a known ATM inhibitor, is well tolerated even with chronic consumption, ATM inhibition could be an interesting new target for the treatment of HD.

\section{Alexandra Flemming}

ORIGINAL RESEARCH PAPER Lu, X.-H. et al. Targeting ATM ameliorates mutant Huntingtin toxicity in cell and animal models of Huntington's disease. Sci. Transl. Med. 6, 268ra178 (2014) 